Théologiques

Théologiques

\title{
Je crois en Dieue... La théologie féministe et la question du pouvoir
}

\section{Louise Melançon}

Volume 8, numéro 2, automne 2000

Le pouvoir

URI : https://id.erudit.org/iderudit/005016ar

DOI : https://doi.org/10.7202/005016ar

Aller au sommaire du numéro

\section{Éditeur(s)}

Faculté de théologie de l'Université de Montréal

\section{ISSN}

1188-7109 (imprimé)

1492-1413 (numérique)

Découvrir la revue

\section{Citer cet article}

Melançon, L. (2000). Je crois en Dieue... La théologie féministe et la question du pouvoir. Théologiques, 8(2), 77-97. https://doi.org/10.7202/005016ar
Résumé de l'article

En « féminisant » Dieu, la théologie féministe se trouve à confronter lepouvoir masculin et patriarcal comme pouvoir de domination. Dans cetarticle, nous donnons un aperçu d'études sur le patriarcat, particulièrementdans les religions, afin de cerner l'origine et donc les conditions danslesquelles le pouvoir patriarcal s'est développé. Puis dans la présentation desdiscours féministes sur Dieu, dans une perspective critique d'abord, et dansun effort de reconstruction au féminin ensuite, apparaissent des modèles derapports de pouvoir, tout autant qu'une conception du pouvoir. Unquestionnement de fond suit qui engage la poursuite d'une réflexionarticulée sur des pratiques de dire Dieue. 


\title{
Je crois en Dieue... \\ La théologie féministe et la question du pouvoir
}

\author{
Louise MELANÇON \\ Faculté de théologie, \\ d'éthique et de philosophie \\ Université de Sherbrooke
}

Que la référence à « Dieu ${ }^{1}$ se fasse dans un contexte de pouvoir, il n'y a rien là de surprenant puisque le pouvoir est une réalité humaine comme toutes les autres, au même titre que le désir, la peur, ou l'amour. Plusieurs discours théologiques de la fin $\mathrm{du} \mathrm{XX}^{\mathrm{e}}$ siècle ont exprimé la défaite de l'illusion qui veut que les langages religieux échappent au monde réel. Quand les êtres humains se représentent "Dieu ", il devient un objet humain et culturel, comme les autres, même si c'est juste pour nommer une quête de transcendance ou une foi en l'Invisible.

La plupart des théologies féministes ont, à cet égard, suivi l'exemple des théologies de la libération, pour dénoncer les effets négatifs d'un discours religieux produit dans le contexte d'un pouvoir oppresseur et proposer une ou des images plus libératrices de «Dieu ». Dans les pages qui suivent, je ferai en sorte de montrer quelle conception du pouvoir se trouve mise en lumière par les discours sur Dieu venant des théologies féministes. Pour ce, après quelques préalables (I) concernant la notion de pouvoir, j'aborderai (II) la critique féministe du pouvoir patriarcal social et religieux ; puis, je ferai voir (III) l'application qui en est faite dans les théologies féministes, quand elles réfléchissent sur l'objet "Dieu ", que ce soit dans un discours critique ou dans un essai de re-nommer ou de re-présenter le divin de manière positive

1. J'indique ici par les «» le mot qui renvoie à la Réalité mystérieuse. 
pour les femmes. Je terminerai (IV) par quelques prolongements en forme de questionnement.

\section{Quelques préalables sur la notion de pouvoir}

\section{Notions séculières}

Le pouvoir est d'abord et avant tout une capacité d'agir, une faculté de faire quelque chose, une habileté qui permet d'atteindre des buts. Idéalement, ce serait une force qui agirait en réciprocité, une énergie de collaboration entre les divers membres ou acteurs d'une société. On y a joint couramment la notion d'autorité. Dans les faits, cette réalité s'est inscrite dans une organisation sociale de type hiérarchique qui « autorisait » un chef, roi, empereur, seigneur ou gouvernement, à exercer son pouvoir en restreignant plus ou moins celui des autres. Il s'agit alors d'un pouvoir de domination sur les autres. Le pouvoir peut cependant s'appuyer sur un ascendant, un prestige, un charisme qui agit par influence plutôt que par des moyens inscrits dans une institution et par des lois. On pourrait alors parler d'un pouvoir non formel accompagné d'une autorité morale qui agit non moins réellement que le pouvoir institutionnel.

\section{Notions religieuses}

Dans le champ religieux, cette réalité du pouvoir existe tout autant, fonctionne de la même manière, mais s'appuie généralement sur une croyance en un Être dont la puissance est absolue. Ce Tout-Puissant donne ou confère des pouvoirs qui permettent à certaines personnes de participer à sa puissance à divers degrés, et ainsi d'agir en son nom $^{2}$. Il y a alors "sacralisation » du pouvoir. Dans le judaïsme, la puissance du Dieu Créateur a été expérimentée d'abord en relation avec des actes libérateurs pour le peuple. Seul Dieu est puissant. L'autorité humaine est subsidiaire à celle de Dieu et conçue comme un service. Elle fut d'ailleurs peu à peu établie en référence à la sagesse plutôt qu'à la puissance des armées. Quant au christianisme, il est né d'une expérience de foi en Jésus, le Serviteur de Dieu, qui s'est abandonné dans l'impuissance sur une croix.

2. Du moins dans les religions théistes ou monothéistes. 
Cependant, assez rapidement, les chrétiens ont dû se situer par rapport au pouvoir politique. Chez les Pères de l'Église, on remarque aisément l'influence de la politique romaine : "La régulation de l'unité par la centralisation a influencé d'abord au niveau biblique et pastoral, mais bientôt également au niveau juridique, la théorie de l'autorité de l'évêque ${ }^{3}$ ». Ignace d'Antioche, le premier, exprimera une vision centraliste et cléricale de l'Église, en faisant de l'évêque le représentant du Dieu unique. Cette conception hiérarchique au service de l'unité harmonieuse des composantes de la société donnera lieu à la théorie des deux pouvoirs, le temporel et le spirituel, qui, en Occident, présidera au développement du christianisme.

Par contre, la critique des pouvoirs injustes déjà très présente dans le judaïsme par l'intermédiaire des prophètes, et ensuite dans la vie de Jésus, continuera de se faire de diverses manières, au cours des siècles. La vie monastique, particulièrement selon Benoît, proposera un modèle de réforme de l'Église, en ce que l'abbé n'est pas un seigneur, ni un évêque, et que tous sont frères — ou sœurs — au sein d'une communauté. Mais jusqu'à nos jours, cela restera un signe de contestation, plus ou moins visible, et sans réel effet sur le modèle ecclésiastique.

\section{Critique féministe du pouvoir patriarcal}

\section{Dans les sociétés}

Les discours féministes, surtout au cours des années 1960-70, ont mis en lumière, en le qualifiant de " patriarcal », le pouvoir exercé sur les femmes dans nos sociétés. On dénonçait pour le moins une asymétrie $\mathrm{du}$ pouvoir entre les hommes et les femmes, sinon un pouvoir de domination jugé destructeur et oppresseur ${ }^{4}$. Au départ, dans le sens strict, le patriarcat veut dire " la règle du père " : il s'agit d'un système de relations sociales où les chefs de famille, des mâles, règnent sur l'ensemble des personnes dépendantes qui en font partie. À l'époque classique, par exemple, dans les sociétés hébraïques, grecques et romaines, les personnes dépendantes sont les épouses, les filles non

3. Nouveau dictionnaire de théologie, Paris, Cerf, 1996, p. 60, $2^{\mathrm{e}}$ col.

4. Dictionary of Feminist Theologies (Letty Russell \& J. Shannon Clarkson editors), Westminster John Knox Press, Louisville, Kentucky, 1996, p. 220. 
mariées, les fils encore dépendants, et les esclaves aussi bien hommes que femmes. Le paterfamilias possède ainsi sa famille comme il possède sa terre et son troupeau. Mais alors que les fils deviendront autonomes et que même les esclaves masculins pourront être affranchis, les femmes, comme épouses, filles ou veuves, seront soumises au nom de leur sexe et les femmes esclaves pourront être abusées physiquement et sexuellement, au nom de cette propriété patriarcale ${ }^{5}$.

Ce système de domination a pris place, dans l'histoire humaine, il y a des millénaires. De plus en plus d'études archéologiques, ethnologiques et anthropologiques arrivent à situer l'origine du patriarcat, de manière à la considérer comme un fait historique et non une "chose naturelle ». Les principales hypothèses indiquent le passage du monde de l'agriculture à celui de l'urbanisation, les déplacements des populations et les invasions, comme étant déclencheurs à la fois des guerres et de la montée du patriarcat ${ }^{6}$. Ce système a certes connu une évolution, pris diverses formes, selon les lieux géographiques ainsi que les contextes sociaux et culturels. Dans nos sociétés modernes, suite aux luttes et révolutions socialistes et à l'arrivée de régimes politiques plus démocratiques, les femmes ont pu sortir de leur exclusion et obtenir des droits égaux à ceux des hommes. Mais ce mouvement, plutôt récent dans l'histoire humaine (les $\mathrm{XIX}^{\mathrm{e}}$ et $\mathrm{XX}^{\mathrm{e}}$ siècles), n'a pas encore atteint tous les coins du monde.

Et même dans des sociétés dites avancées comme les nôtres, les femmes continuent d'être des travailleuses domestiques non rémunérées et les premières responsables de l'éducation des enfants. Dans le monde du travail aussi, malgré des progrès certains, leur accès à l'égalité reste encore limitée.

Cependant, peu à peu, à l'intérieur même du mouvement des femmes, des malaises, des contestations et des divisions révèlent la présence du pouvoir de domination chez les femmes elles-mêmes. Les différences de classes sociales et de races existent aussi parmi les femmes de telle façon que dans une société où les hommes blancs dominent, les femmes blanches participent à ce pouvoir sur les femmes

5. Idem, op. cit., p. 205.

6. Dans son introduction à Women in World religions (ed. Arvind Sharma), State University of New York Press, 1987 : Katherine K. Young se réfère à deux ouvrages auxquels nous reviendrons plus loin. 
d'autres races ou couleurs. Une révision de l'analyse féministe du pouvoir patriarcal a suivi cette prise de conscience venue de l'interpellation de celles qui sont victimes, à divers titres, de l'oppression patriarcale. On a remis en question la manière essentialiste et dualiste de concevoir le patriarcat comme un pouvoir des hommes sur les femmes, en fonction du genre seulement, et comme si celles-ci n'étaient que des individus et non des membres de la société 7 .

De même, avant le patriarcat, il n'y avait pas une situation idyllique, comme certaines féministes l'ont parfois laissé entendre. Le pouvoir des femmes pouvait être prééminent dans les sociétés primitives, bien que ce qu'on appelle le " matriarcat " n'est pas à comprendre comme l'équivalent du patriarcat. Le pouvoir des femmes de porter un enfant, d'en accoucher, de l'allaiter fut sans doute considéré comme un énorme pouvoir, un pouvoir sacré, pouvoir sur la vie et la mort, et sans doute aussi comme un pouvoir menaçant à l'égard des hommes. Mais en même temps, ces fonctions maternelles limitaient les actions des femmes ${ }^{8}$.

Le mouvement moderne des femmes, en voulant déconstruire les relations de pouvoir pour transformer les valeurs et institutions sociales, a mis de l'avant une conception du pouvoir qui vient de l'intérieur, qui permet aux femmes de reconnaître et d'apprécier leurs talents et responsabilités, un pouvoir ( "empowerment ") qui consiste à développer sa force et son influence pour son bien-être et celui de tous, au plan personnel, interpersonnel autant que socio-économique et politique. Au lieu d'être un moyen de contrôler les autres, de leur enlever leur propre pouvoir, cette conception de l' " empowerment » donne d'agir avec efficacité dans une perspective de transformation, par la collaboration avec les autres. C'est une énergie créatrice qui nourrit, qui accomplit les personnes tout autant qu'elle combat les oppressions ${ }^{9}$.

En dévoilant la participation des femmes au pouvoir de domination, la réflexion féministe en est ainsi venue à développer l'intégration du pouvoir et de la différence. Cette compréhension amène une

7. Dictionary of Feminist Theologies, op. cit., p. 220.

8. Dictionary of Feminist Theologies, op. cit., p. 220.

9. On a compare cette énergie au pouvoir érotique. Cf. la poète afroaméricaine Audre Lorde, dans Dictionary of Feminist Theologies, op. cit., p. 83-84. 
reprise de la solidarité entre les femmes, nommée la sororité, dans une perspective de mutualité et de relationnalité.

\section{Dans les religions}

Si le patriarcat est une réalité sociale née à un moment particulier de l'évolution des sociétés humaines, il a été en même temps une réalité religieuse. La majorité des religions sont nées et se sont développées dans le contexte du patriarcat ${ }^{10}$. Mais si elles ont des traits communs concernant la subordination des femmes, elles comportent aussi des différences ${ }^{11}$.

L'autorité masculine et le pouvoir ne concordent pas toujours. Par exemple, même si l'homme représente l'autorité dans la famille, c'est la femme qui a le pouvoir réel dans le domaine domestique. Ou les femmes n'ont pas la même référence religieuse que les hommes. $\mathrm{Ou}$ bien encore, si les femmes sont exclues du domaine religieux, c'est pour d'autres raisons que la misogynie, et parfois, il y a une exclusion parallèle des hommes par rapport à certaines activités religieuses. Cette variété de situations observables au cours des siècles, selon les lieux géographiques ou les contextes culturels, a donné lieu à des représentations variées de la transcendance : dieux, déesses et êtres androgynes, dans un être unique ou dans une dualité, ou encore comme ce qui est au-delà de toute forme, tout genre ou tout nombre.

Cependant, il y a des traits communs à toutes les religions patriarcales. Dans les sociétés tribales, on organisait les rôles sexuels généralement de manière harmonieuse, dans la complémentarité des différences. Le patriarcat a élargi l'écart entre les sexes. Des recherches $^{12}$ se sont poursuivies à partir de l'hypothèse que la domination masculine s'est développée dans les religions tribales dans des conditions précises, à savoir : une ouverture sur l'extérieur, la séparation

10. Elizabeth A. JOHNSON, Dieu au-delà du masculin et du féminin (trad. de l'américain de She Who Is), Cogitatio fidei 214, Cerf, Paris 1999, pp 398-410. 11. L'ouvrage mis en référence plus haut, édité par Sharma et introduit par K. Young, Women and World Religions, est une source intéressante pour traiter de cette question.

12. K. Young, op. cit.. Peggy Reeves Sanday avance cette explication dans son livre Female Power and Mal Dominance: On the Origins of Sexual Inequality, London, Cambridge University Press. 
des sexes et une situation de stress. Que ce soit la chasse aux grands animaux ou la guerre contre des envahisseurs, ces situations auraient exacerbé la séparation des sexes, contrairement à une symétrie des rôles dans les tribus primitives en temps de paix. Selon Élie Sagan ${ }^{13}$, le développement d'une domination masculine extrême (qu'on remarque d'ailleurs dans la période de formation du judaïsme, du brahmanisme et du confucianisme) serait relié à une situation de stress en temps de guerre et ensuite, au moment de la construction des royautés ${ }^{14}$. Ces sociétés développent un système social ordonné de manière hiérarchique et une orientation religieuse de type sacrificiel ${ }^{15}$.

Sagan compare ce phénomène social à celui du développement de l'enfant, selon la perspective psychanalytique. L'enfant doit, pour accéder à son individualité, se séparer de sa mère. Cela se fait dans l'ambiguité et l'anxiété ; il y a à la fois la peur d'être englouti dans la mère et l'insécurité de se trouver séparé d'elle. Pour résoudre son conflit, l'enfant se tournera vers le père ${ }^{16}$. Dans les sociétés tribales, les rites de l'initiation des garçons jouaient ce rôle. À l'époque des guerres, du développement des royautés, la solidarité masculine emprunte le modèle violent de domination du roi sur les autres pour soumettre les femmes sur lesquelles on projette la menace du pouvoir maternel :

...the male bonding that ultimately brings us tyranny against children, women, and deprived classes has its genesis at this moment. Fearing the mother's power to re-engulf not only their individuality but also their unique maleness, fathers and sons join together to make females harmless through degradation... ${ }^{17}$

13. K. YounG, dans le livre ci-haut cité, utilise aussi les thèses de Élie Sagan, dans At the Dawn of Tyranny: the Origins of Individualism, Political Oppression, and the State, New York, Alfred Knopf.

14. Ibid., p. 9 : "Sagan's thesis is that societies pass through a state of development from the social cohesion provided by kinship and the confederation of tribes to a centralized monarchy, which is organized by loyalty to the king on fear of his power to oppress."

15. Selon Sagan, on trouve l'équivalent dans les traditions: indiennes, polynésiennes ou africaines.

16. Il s'agit ici de l'enfant mâle. K. Young fait la remarque que la fille, demeurant dans le domaine de la mère, ne suit pas la même évolution. $\mathrm{C} f$. op. cit. p. 13.

17. K. YounG, op. cit., p. 12-13. 
Les femmes, sans la sphère domestique, n'ont pas à se battre ; elles se consacrent à la vie. Les hommes, pour défendre la vie, tuent. Ils peuvent éprouver de l'envie pour le rôle des femmes dans la protection de la vie ; aussi la patrilinéarité leur permettra d'avoir le contrôle sur la famille. Cette asymétrie des rôles dans la subordination des femmes permet une certaine complémentarité : les femmes, en retour, sont protégées physiquement et économiquement. Cependant, le contrôle absolu sur les femmes comprenait la monogamie pour celles-ci, alors que les hommes, eux, jouissaient de la polygamie et de la prostitution : "this split in attitudes of women and the tension between chastity and sexual license characterize much of the history of patriarchal religions ${ }^{18}$ ».

Le développement d'un tel système de domination patriarcale a eu des conséquences sur la représentation de la déité notamment celle-ci: la transcendance est vue en dehors du cosmos. Dans une monarchie absolue, "Dieu » est le Tout-Puissant même s'il garde et protège son peuple, comme un Père. Dans les religions universelles, Il est le Créateur qui domine sa Création ; mais par rapport aux religions éthiques, l'accent sera mis sur l'individu et son expérience plutôt que sur le corps social, lequel est fondé sur un corps doctrinal. Par ailleurs, la plupart des religions ont une sotériologie inclusive : elles prônent l'égalité des hommes et des femmes. Ce qui explique que malgré la domination d'un Dieu mâle, les femmes ont pu vivre et exprimer leur spiritualité.

\section{Dans le christianisme}

L'histoire du christianisme est traversée par un conflit entre deux visions : l'une qui affirme l'égalité de l'homme et de la femme par rapport au salut, et l'autre qui définit les femmes comme subordonnées aux hommes, aussi bien sur le plan ontologique que sur le plan social ${ }^{19}$. Héritière des religions patriarcales, des modèles culturels venant des philosophies grecque et romaine aussi bien que de la loi juive, la religion chrétienne aura une influence décisive sur l'Occident autant pour la situation des femmes que pour la conception de «Dieu ».

18. K. YounG, op. cit., p. 15.

19. Rosemary RADFORD RUETHER, Christianity, dans K. YOUNG, op. cit., p. 208 ss. Cf. de son ouvrage: Women and Redemption. A theological History, Fortress Press, Minneapolis, 1998. 
Cette double vision, qu'on trouve dans des textes de Paul relisant la Genèse, s'est articulée dans une théologie de la subordination où la domination masculine correspond à l'ordre de la création, à la volonté de Dieu, alors que, dans la perspective du salut, les femmes en sont l'objet, à condition de sortir de leur condition "d'Ève pécheresse», d'être des mères soumises à la loi de leurs pères et maris, ou des "vierges » vivant comme des hommes, c'est-à-dire en dehors de leur condition d'épouse et de mère selon la chair. Ce dualisme, qui s'ajoute à la vision conflictuelle, s'appuie sur la foi en un "autre monde " supérieur à celui-ci. Augustin ira même jusqu'à dire que la femme, à cause de son corps, n'est pas à l'image de Dieu comme l'homme ; et elle ne peut donc accéder "aux ordres sacrés » pour représenter le Christ. Au Moyen Âge, Thomas d'Aquin, en reprenant Aristote, donnera un appui à l'infériorisation des femmes ; il les trouvait biologiquement "défectueuses " par rapport à la semence mâle. La subordination des femmes au pouvoir masculin est ainsi fortement ancrée dans le christianisme et la dévaluation de l'être féminin s'ajoute à son infériorisation sociale. La domination du pouvoir religieux, sacré dans le christianisme, est dorénavant bien établie. Les mouvements marginaux, d'ailleurs vite jugés non orthodoxes, seront des lieux sans influence pour le pouvoir féminin. Et la vie monastique elle-même, malgré ses résistances, sera bien encadrée par le pouvoir patriarcal.

Cette théologie de la subordination des femmes sert de modèle pour celle concernant les esclaves, ou les autres races. Elle révèle et justifie à la fois une conception patriarcale de " Dieu ».

God is typically imaged as a patriarchal father and Lord. The patriarchal hierarchies of male over female, father over children, and master over slave are reduplicated symbolically in the relation of God and Christ to the Church, as father to sons, lord to servants, and bridegroom to bride. The image of Christ as head of the church, which is his body reduplicates the legal view in which the wife lacks her own "head" (selfdirection) and belongs as body to her husband. ${ }^{20}$

Pourtant, l'expérience originaire décrite dans les évangiles semble plus proche d'un autre modèle. Jésus avait des affinités avec le mou-

20. Rosemary. R. RUETHER, Dictionary of Feminist Theologies, op.cit., p. 206. 
vement prophétique pour annoncer une bonne nouvelle aux pauvres, contester l'exclusion des marginaux, particulièrement les femmes, de la religion juive et patriarcale de son temps. Il défait l'absolu des hiérarchies : le premier sera le dernier, le maître essuie les pieds de ses disciples... Il rétablit les êtres dans leur dignité : les enfants, les prostituées... Il partage sa table avec tous - ou s'invite à la table des pécheurs... Il parle d'un Père-Abba, tout proche, dans ce contexte de tendresse et de compassion pour tous... Ses disciples vivent dans son intimité, aussi des femmes... non seulement il les initie à ses pouvoirs, il en fait aussi ses amis et amies... Il relativise la famille et permet à des femmes de sortir de la sphère domestique.

Mais un mouvement de "patriarcalisation » va par la suite remplacer le "mouvement de Jésus », comme l'a montré SchüsslerFiorenza $^{21}$ : ce qui a "couvert " la réalité d'une ekklésia à l'origine bien autre que l'assemblée élitiste de la démocratie grecque ou bien autre que l'Église masculine et dominante, celle des églises domestiques des premiers chrétiens et chrétiennes.

\section{Quand des femmes disent Dieue}

\section{Au-delà du Dieu Père}

Au début des années 1970, quand Mary Daly ${ }^{22}$ titrait son livre, elle s'inscrivait dans un tournant (plus) radical du mouvement des femmes. Jusqu'à ce moment, les femmes travaillaient à réclamer la justice et l'égalité par la revendication de leurs droits, l'accession au monde des hommes. Il s'agissait maintenant d'aller aux causes, aux racines du mal : le sexisme inscrit dans le système patriarcal qui se transmet, se maintient par le processus de socialisation des rôles sexuels. Et la religion patriarcale fait partie des idéologies qui cachent la réalité de " la caste sexuelle » en prétendant que c'est naturel, que c'est conforme à la

21. Elisabeth SCHÜSSLER-FIORENZA, En mémoire d'elle (trad. de l'américain), Cogitatio Fidei 136, Cerf, Paris, 1986. Plus tard, SchüsslerFiorenza parlera de "kyriarchie " dans Jesus, Myriam's Child, Sophia's Prophet, Continuum, New York, 1994, p. 12 ss.

22. Beyond God the Father. Toward a philosophy of women's liberation, Beacon Press, Boston, 1974. 
volonté divine ${ }^{23}$. Pour Mary Daly, cette prise de conscience exige du courage de la part des femmes parce qu'elles doivent sortir de leur aliénation :

This is the courage to see and to be in the face of the nameless anxieties that surface when a woman begins to see through the masks of sexist society and to confront the horrifying fact of her own alienation from her authentic self (p. 4).

Démasquer cette situation et travailler sur les représentations, les symboles, les langages, voilà ce qui permettra aux femmes de se réapproprier leur pouvoir comme être humain, à savoir : se nommer, nommer le monde et "Dieu " à partir de leurs expériences plutôt que d'être soumises au langage androcentrique et aux représentations sexistes du monde patriarcal. Le mouvement des femmes deviendra révolutionnaire plutôt que réformiste. La théologienne Daly sera au départ d'une entreprise intellectuelle qui n'a pas cessé depuis : celle de dire «Dieu » de manière libératrice pour les femmes. Pour sa part, elle dénoncera l'image du Dieu Père comme étant la projection des représentations patriarcales, comme étant en fait une "idole ». On dit que Dieu a créé l'homme à son image, mais en réalité, les hommes ont créé Dieu à leur image : "Si Dieu est masculin, le mâle est Dieu » (p. 191).

Le travail théologique comprend d'abord une tâche d'iconoclaste ; il faut briser les idoles et ensuite, tendre à aller au-delà de nos projections. Si nous avons l'intuition d'être « à l'image de Dieu ", n'est-ce pas par notre pouvoir créateur ? : "It is the creative potential itself in human beings that is the image of God» (p. 29). Ainsi, nous devenons humains en faisant l'expérience de la transcendance. Le monde patriarcal a pensé la transcendance en dehors du monde : Dieu est un Etre suprême. Les femmes qui participent à leur révolution, à leur libération, peuvent créer un nouveau contexte pour penser et dire Dieu autrement. Du moins elles peuvent poser de nouvelles questions : "Why indeed must 'God' be a noun ? Why not a verb - the most active and dynamic of all? » Et la réponse pourrait être : "God is a Verb... God is BEING ». Dieu est un processus dynamique, une puissance créatrice dans laquelle nous participons aussi par notre créativité.

23. Ibid., p. 2-3. 
Les femmes en se libérant, en s'affrontant à leur aliénation, participent de ce mouvement créateur et deviennent des êtres authentiques au lieu d'être aliénées sans identité propre. En cherchant à sortir du monde patriarcal, elles se créent une nouvelle vie et ainsi, contribuent à un avenir pour toute l'humanité : elles participent du pouvoir de transformation de Dieu qui fait toutes choses nouvelles (p. 43).

\section{2. À la recherche de "Déesse »}

Dans la foulée du tournant révolutionnaire dont je viens de parler, est apparue dans les discours féministes la référence à «Déesse ». Cette référence féminine dans les discours théologiques se présente sous bien des formes, comme nous allons le voir, mais c'est un mouvement global dont la caractéristique principale est de reprendre la question de genre qui avait été délaissée dans ce qu'on a appelé le féminisme égalitaire. Élisabeth Parmentier ${ }^{24}$ parle de l'enjeu de ce "féminisme gynécentrique » de la façon suivante :

...Il s'agissait de réintégrer le corps et la « nature » féminine, qu'il avait fallu occulter dans les combats égalitaires, sans pour autant retomber dans les travers d'autrefois concernant la vocation spécifique de la femme... C'est une véritable contre-culture, un contre-pouvoir, une her-story, une histoire au féminin, que s'élaborait en prenant appui sur les goûts traditionnellement féminins : le mystère, l'irrationnel, la recherche spirituelle.

La théologie qui se développe ainsi s'appuie sur les expériences des femmes comme femmes pour les mettre en relation avec le divin, ou la déité, ou le sacré. Ce faisant, elle redonne aux femmes leur pouvoir dans le domaine spirituel ou religieux, pouvoir qui leur avait été enlevé ou dont elles avaient été privées depuis bien des siècles. Ce pouvoir est lié à la sortie de leur aliénation sociale, économique, politique et spirituelle en même temps qu'à la valorisation et à l'épanouissement de leur genre ${ }^{25}$.

24. Élisabeth PARMENTIER, Les filles prodigues. Défis des théologies féministes, Labor et Fides, Genève, 1998, 279 p. Ce livre à perspective œcuménique a, pour moi, le grand mérite de rendre compte de la discussion féministe dans les milieux européens et même au niveau mondial.

25. Mary Daly, avec son livre Gynaecology, Beacon Press, Boston, 1987, et les autres qui vont suivre, emprunte cette voie, en privilégiant le travail sur le langage. 
Ce mouvement tracera diverses voies qui explorent non seulement une variété d'images et de représentations du divin, mais aussi des différences de vision de notre rapport au divin ${ }^{26}$. L'utilisation du mot "Déesse » peut renvoyer aux religions polythéistes où existaient les cultes aux déesses : certaines féministes retournent à ces religions anciennes pour vivre leur dimension spirituelle. D'autres considèrent "Déesse » comme la déité qui était invoquée, avant le patriarcat, aussi bien par les hommes que par les femmes : Cette Grande Déesse ${ }^{27}$ renvoie davantage à la " mater ", à la déité matricielle d'où vient tout ce qui existe. De leur côté, les théologiennes juives et chrétiennes trouvent facilement des noms ou attributs féminins pour " Dieu ", à l'intérieur même de leurs traditions, qu'elles soient marginales ou officielles ${ }^{28}$. Elles peuvent ainsi avancer l'idée d'un Dieu-mère aussi important que le Dieu-Père. Pour d'autres, elles proposent des images nouvelles comme l'Ami/e, l'Amant/ $\mathrm{e}^{29}$, ou reprennent de manière neuve des noms divins, comme l'Esprit ${ }^{30}$. C'est ainsi que les discours féministes religieux sont traversés de divergences importantes, comme le polythéisme ou le monothéisme, l'exclusivité féminine ou l'inclusion du genre dans la déité et l'usage de la Déesse comme métaphore pour dire le Divin plutôt que comme déité31.

Ces représentations variées cherchent toutes à libérer les femmes dans leur relation au sacré, ne serait-ce qu'en les enracinant dans leurs expériences de femmes, mais elles ne réussissent pas toujours à donner un réel pouvoir par rapport à la domination masculine, ne serait-ce que parce qu'elles gardent un modèle stéréotypé de séparation des rôles. Et surtout, elles ne contribuent pas nécessairement à remettre en cause une conception de la divinité qui est à la base des pouvoirs de domination.

26. Dictionary of Feminist Theologies, op. cit., p. 110, note 3.

27. Merlin Stone, Quand Dieu était femme, éd. L'Étincelle (trad. de l'américain), Montréal, 1979.

28. Élisabeth. PARMENTIER, op. cit., p. 110, note 3.

29. Cf. par exemple, Sallie McFAGUE, Models of God: Theology for an Ecological Nuclear Age, Philadelphia, Fortress Press, 1987.

30. Cf. par exemple, Elizabeth A. JOHNSON, Dieu au-delà du masculin et du féminin (trad. de l'américain She Who Is), Paris, Cerf (Cogitatio Fidei 214)1999, ch. VII.

31. Dictionary of Feminist Theology, op. cit., p. 131. 


\section{Quel pouvoir au féminin?}

Est-ce que féminiser «Dieu » accorde réellement du pouvoir aux femmes ? Cette question fort pertinente est traitée par des théologiennes ${ }^{32}$ comme Rosemary Radford Ruether ou Élizabeth Johnson qui montrent l'ambiguité ou même l'insuffisance de certaines manières de procéder.

On peut garder un modèle de subordination au masculin en attribuant des traits féminins à Dieu, comme ceux qui relèvent des rôles traditionnels des femmes, une maternité idéalisée, par exemple, pour tempérer des traits masculins trop violents :

L'introduction de caractéristiques présumément féminines s'opère à l'intérieur d'une configuration androcentrique, laissée intacte. Puisque l'image de Dieu correspond toujours à celle du pouvoir masculin, même si elle est figurée en des teintes plus douces, le féminin se trouve intégré, de manière subordonnée, à un symbole global qui demeure masculin. ${ }^{33}$

Les qualités dites féminines servent à parler d'un Dieu bon plutôt que sévère, et cela peut justifier de manière plus subtile le pouvoir de domination des hommes, en l'accolant au "paternalisme ». On peut dire que Dieu agit comme une mère, mais on trouve scandaleux de dire qu'il est une mère. De même on peut faire voir des dimensions féminines en Dieu sans sortir des stéréotypes du masculin et du féminin ${ }^{34}$. Le problème réside dans le fait qu'on attribue du "féminin » aux hommes mais pas de traits "masculins » aux femmes. C'est toujours un modèle inégalitaire qui est sous-jacent. Cette "simplification dualiste" a déjà été dénoncée par R. Radford Reuther ${ }^{35}$ pour qui des traits culturels féminins, comme on en trouve d'ailleurs dans la Bible, sont le produit d'une structure de domination. Pour que ces images féminines soient libératrices pour les femmes, il faut les situer dans notre contexte où les rôles sexuels ne sont plus rigi-

32. Je m'arrêterai ici aux réflexions faites par des théologiennes de tradition judéo-chrétienne, même s'il m'apparaît important, par ailleurs, d'avoir une vision plus globale de cette question.

33. Elizabeth JOHNSON, op. cit. p. 81-82. Elle fait remarquer que c'est souvent des théologiens masculins, mais pas uniquement, qui proposent ainsi d'adoucir le "Dieu patriarcal ».

34. C'est souvent, aujourd'hui, en s'appuyant sur l'apport, par ailleurs intéressant de Jung... cf. la critique de Naomi Goldenberg citée par E. JOHNSON, op. cit., note 20, p. 83.

35. La féminité de Dieu, Concilium 163, 93-101. 
des, et où la diversité et les différences qu'elles soient biologiques ou culturelles, reposent quand même sur un fondement égalitaire.

C'est ainsi que des théologiennes mettent de l'avant un modèle d'équivalence dans la reconstruction d'un discours théologique au féminin. Cela implique l'utilisation de l'analogie, comme l'a développé la théologie classique, mais à partir des expériences des femmes comme étant aussi valables que celles des hommes.

Johnson le fait de manière remarquable à partir de l'expérience de l'Esprit, et dans la perspective chrétienne d'un Dieu trine. Cela l'amène à parler de l'Esprit au féminin (Esprite $\left.{ }^{36}\right)$, non comme subordonnée au Père et au Fils, comme l'ont fait bien des interprétations de la Trinité, mais en rapport avec l'action de Dieu dans le monde, action créatrice représentée dans la Bible par la Sagesse (Sophia). Ce faisant, elle déploie de nouvelles interprétations théologiques qui concernent la définition de Dieu pour tous les croyants et croyantes. L'inclusion du féminin de manière équivalente concourt au renouvellement de la théologie, en plus de fonder le pouvoir propre des femmes :

Une démarche partant de l'expérience de l'Esprit, reconnue au fil d'une interprétation, et se poursuivant en une réflexion qui aboutit à Dieu vivante, sur la Trinité vivante, offre une voie permettant d'engager un propos à la lumière des insights déclenchés par l'expérience religieuse marginalisée des femmes, une voie qui, au surplus, fait ressortir bien des réalités ignorées par l'approche déductive. ${ }^{37}$

En parlant de l'Esprit-Sophia, de Jésus-Sophia, de Mère-Sophia (p. 203-297), Johnson explore une figure féminine très importante dans la tradition judéo-chrétienne pour parler du Mystère divin ${ }^{38}$. Les métaphores féminines mettent en lumière l'action créatrice et libératrice de la Sagesse telle que manifestée dans l'histoire du prophète Jésus pour nous relier à "Celle qui est ${ }^{39}$, Dieu vivante ${ }^{40}$, créatrice et

36. Élisabeth PARMENTIER, dans le livre déjà cité traduit ainsi l'usage du féminin pour l'Esprit que Johnson féminise par le "she » ou le " her ".

37. Elizabeth JOHNSON, op. cit. p. 200-201.

38. Johnson n'est pas la seule. En particulier, avant elle, E. SchüsslerFiorenza l'avait fait dans son livre Jesus, Myriam's child and Sophia's Prophet. 39. Elizabeth JOHNSON, ibid., p. 373 ss.

40. Le traducteur du livre de Johnson (Pierrôt Lambert) écrit aussi Dieu au masculin, accolé d'un adjectif féminin. 
recréatrice, la matrice de tout ce qui existe. Ainsi, l'inclusion du féminin dans le discours sur "Dieu " ne renvoie pas "à une dimension féminine du divin, mais exprime en une image féminine l'intégralité de la puissance et de la sollicitude divine ${ }^{41}$. Comme les métaphores masculines, les féminines disent le «Dieu » trine chrétien à partir des expériences des femmes jugées aussi valables, en même temps tout autant relatives que celles des hommes. Par contre, le fait de parler du Mystère à l'origine comme « Mère » ou " matrice » ne privilégie pas la fonction spécifique des femmes - la maternité comprenant la grossesse, l'accouchement et l'allaitement - mais plutôt l'acte d'engendrement où les rôles masculins et féminins sont égaux ou équivalents. On évite ainsi de renverser le modèle patriarcal, centré sur Dieu Père, pour hisser à sa place la mère toute-puissante, la mère archaïque sur laquelle l'enfant peut projeter sa peur d'être englouti.

Par ailleurs, que la Sophia soit reprise pour dire le modèle trinitaire renouvelle d'idée d'un Dieu relationnel et permet de penser la puissance divine comme un pouvoir-de-relation. Les liens entre l'Esprit et la Sagesse, repris dans une interprétation féministe, montrent une conception de l'amour-don autre que celle de la prédication traditionnelle adressée aux femmes, en terme d'oubli de soi, de sacrifice, pour mettre de l'avant l'amour comme " pouvoir qui mobilise toute vie, l'élan qui pousse tout être vivant vers les autres êtres » (p. 232). C'est dans l'horizon de la mutualité, de la réciprocité et de la liberté que naît une conception de la puissance comme énergie transformatrice : " une puissance libre, vitalisante, non violente, qui est source de relations, de régénération, d'épanouissement» (p. 218).

D'un autre côté, quand on traite du pouvoir dans une perspective chrétienne, on ne peut faire autrement que de rencontrer un paradoxe : celui de l'expérience de Jésus de Nazareth qui meurt impuissant devant les pouvoirs religieux et politiques. Les théologies féministes suivent la voie ouverte par les «théologies» du Crucifié ${ }^{42}$ pour contester l'idée théiste d'un Dieu apathique et tout-puissant, et développent une compréhension d'un Dieu qui souffre. D'une part, la perspective féministe valorisant beaucoup la relationnalité, elle

41. Elizabeth JoHnson, ibid., p. 93.

42. Pensons ici à J. Moltmann, J. Jüngel, Dorothee Sölle, et à des théologiens de la libération comme Gutierrez, Sobrino... 
dénonce l'idée d'un dieu apathique comme étant une projection de l'homme en position de domination pour qui " la liberté est synonyme d'autarcie, d'autonomie et de maîtrise ${ }^{43}$ »; puis elles s'appuient sur les diverses douleurs, les détresses et déchéances de toutes sortes vécues par les femmes dans le monde, pour affirmer la possibilité de la douleur dans le mystère divin, et ce, à titre de imago dei, imago Christi ${ }^{44}$. D'autre part, voulant éviter que l'idée "d'une victime divine impuissante » renforce la dépendance des femmes et leur capacité de souffrance passive, elles cherchent à intégrer la démarche de libération entreprise par bien des femmes par leur engagement contre l'injustice et l'expression de leur colère :

Elles mettent en œuvre à cette fin un pouvoir qui est une vitalité, une vigueur dynamisante qui crée des liens et stimule chez elles et autour d'elles la liberté et la force personnelles. Elles déploient une énergie qui interpelle, qui anime, qui éveille, qui avive l'autonomie et l'amitié. Elles activent un mouvement de l'esprit qui est tour à tour mouvement de construction, de réparation, de solidarité, d'opposition, de réjouissance ou d'affliction. Ce mouvement transforme les êtres et crée des relations entre eux et des rapports entre eux et le monde. Ce dynamisme n'est pas l'antithèse de l'amour, mais plutôt la forme que prend l'amour devant les forces du nonêtre et de la mort. Ce dynamisme opère relationnellement ${ }^{45}$.

Aussi l'image de la Sagesse qui partage tout de l'humain ne s'oppose pas à son pouvoir de compassion : "Sophia-Dieu est en rapport de solidarité avec les êtres qui souffrent... le pouvoir de l'amour compatissant de Dieu pénètre la détresse du monde pour la transformer de l'intérieur... ${ }^{46}$...il signifie le pouvoir qu'a l'amour souffrant de résister au mal, de recréer le monde ${ }^{47}$ ».

Le travail de féminisation des images, figures, attributs qui réfèrent à « Dieu » est important pour valoriser l'expérience religieuse et spirituelle des femmes. Comme on vient de le voir, c'est un point de départ pour inclure le féminin dans l'expérience humaine la plus éle-

43. Elizabeth JOHNSON, op. cit. p. 391.

44. Ici il faut référer à la thématique de Christa très présente chez les théologiennes chrétiennes.

45. Elisabeth. JOHNSON, op. cit. p. 417.

46. Ibid., p. 419.

47. Ibid., p. 420. 
vée, de telle manière que cela contribue à renouveler pour tous et toutes notre compréhension du rapport au divin. Aussi le fait de garder le mot «Dieu » en lui accolant des pronoms ou adjectifs féminins ne rend pas l'importance du changement que cela suppose. Par contre, l'utilisation de Déesse aussi précieuse soit-elle pour les femmes - et pourrait l'être aussi pour les hommes- ne produit pas le même effet d'inclusion, et renvoie à une époque jugée païenne.

Rosemary Radford Ruether a essayé de mieux exprimer cela en écrivant God/ess pour parler du Mystère comme d'une Réalité unique $^{48}$. En français, dire Dieu/esse est ardu ; mais il pourrait signifier alors le ou la Sans-Nom ${ }^{49}$. Aussi, un groupe de femmes, au Québec, L'autre parole ${ }^{50}$, a choisi d'écrire Dieue. Cette nouvelle pratique de féminisation qui est un choix théologique et politique est un acte local; on ne le trouve pas ailleurs. C'est réellement un acte féministe qui chercher à "sortir Dieu du ghetto masculin ${ }^{51}$. Il indique un pouvoir d'affirmation exercé communautairement et qui favorise la construction d'une identité. Ce sont des femmes situées culturellement, politiquement et qui expriment ainsi leurs expériences diverses comme femmes. Dire « Dieue » est une expérience libératrice, contextuelle, pour des femmes qu'exercent leur pouvoir spirituel, religieux, dans la solidarité et qui forment ekklesia : "Elles deviennent des personnes à part entière et prennent le risque de nommer Dieu à leur façon, avec ce que cela comporte de limites et de vérité ${ }^{52}$.

\section{Quelques questions pour conclure}

Après cette réflexion brève et partielle sur la théologie féministe et le pouvoir, il m'est apparu normal de le faire en termes de questionne-

48. Sexism and God-talk, Beacon Press, Boston, 1993, p. 68-70.

49. Collective de femmes féministes et chrétienne : $c f$. Marie-Andrée RoY et Denise Couture, "Dire Dieue », Dire Dieu aujourd'hui (sous la direction de Camil Ménard et Florent Villeneuve), coll. Héritage et Projet 54, Fides, Montréal, 1994. pp. 139 ss.

50. Ibid. p. 141 ss.

51. Monique DumaIS, "Sortir Dieu du ghetto masculin ", dans Souffles de femmes, sous la direction de Monique Dumais et Marie-Andrée Roy, Éd. Paulines, Montréal, 1989, pp. 135-146.

52. Marie-Andrée RoY et Denise COUTURE, «Dire Dieue ", op. cit., p. 146. 
ment de manière à ce que les limites de ce travail s'ouvrent sur des prolongements complémentaires.

1.

La réécriture au féminin de traditions bibliques ou de traités théologiques a, certes, son importance pour permettre aux femmes de développer leur estime d'elles-mêmes et leur pouvoir spirituel. Mais cela risquerait de ne pas vraiment remettre en question le modèle patriarcal ou de domination masculine, si on ne confrontait du même coup le théisme, le monothéisme ou même l'athéisme (en tant que son opposé) qui projettent Dieu à l'extérieur, comme une entité en dehors de sa création. N'est-ce pas le type de rapport entre le divin ou la Réalité mystérieuse et le monde qui est en jeu quand on parle d'un Dieu qui souffre, par exemple? L'immanence et la transcendance pourraient se penser à l'intérieur d'un processus divin qui change, qui devient avec sa création. La transcendance serait conçue comme une capacité d'aller au-delà d'un état statique, un pouvoir d'une créativité infinie. Ne pourrions-nous pas participer de ce pouvoir? Notre développement ne pourrait-il pas être un "devenir divin ${ }^{53}$ ?

Au lieu de parler de «maternité » qui est une fonction spécifique aux femmes, Jantzen suggère la symbolique de la "natalité ", du fait que nous naissons, et donc que nous recevons la vie et ensuite nous nous développons, avant de mourir comme tout être vivant, que nous soyons femmes ou hommes. L'avantage de cela serait de situer notre finitude dans un processus concret, dynamique plutôt qu'en une catégorie abstraite qui ne fait que " couvrir » notre réalité. Et n'est-ce pas au fond le rapport difficile à la " mater " que tous et toutes ont à vivre puisque nous naissons tous d'une femme ${ }^{54}$ ? Et en même temps, cela exige de considérer la différence des sexes sur cette question, afin d'arriver à un dialogue constructif ${ }^{55}$.

53. Grace M. Jantzen, Becoming Divine. Towards a Feminist Philosophy of Religion, Indiana University Press, 1999.

54. La réflexion écoféministe nous a déjà interpellé/es là-dessus : cf. le livre magistral de R. Radford RuETHER, Gaia and God, Harper, San Francisco, 1992. 55. Plusieurs femme psychanalystes, psychologues se penchent sur cette question du rapport à la mère selon les sexes : ex. Nancy Chodorow, Christiane Olivier, Luce Irigaray, etc. 
2.

Dans cette manière de penser, alors, Dieu n'aurait-il pas un corps? Sa création? Pourquoi l'idée du panthéisme serait à rejeter s'il ne s'agit pas de réductionnisme? N'avons-nous pas l'expérience d'être dans un corps sans être uniquement ce corps? L'altérité a sa place dans cette vision des choses, sans tomber dans un modèle dualiste sous forme de polarisation ou séparation. Au contraire, la diversité, les différences sont affirmées et respectées, contrairement au " monisme » où tout doit être ramené à l'UN. Le phénomène d'objectivité dans la pensée scientifique ne s'enracine-t-il pas aussi dans le même dualisme de séparation hiérarchique que celui de " esprit/corps ", " homme/femme "?

L'intérêt de la prise de parole féministe à ce sujet serait d'obliger à entreprendre la déconstruction du fonctionnement de la raison moderne ${ }^{56}$ de la manière la plus radicale. Ce que les femmes engagées dans un processus de changement apportent de plus neuf, n'est-ce pas ce rapport pratique, concret, incarné avec la réflexion? Avec la théorie? Quand leurs corps individuels et leurs corps communautaires disent dieue, ce n'est pas une projection dans l'idéal abstrait. N'est-ce pas à travers leurs devenirs, leurs histoires, leurs engagements pour la justice et la mutualité ${ }^{57}$ qu'elles participent à la créativité divine? Et aussi leurs solidarités entre elles de diverses situations ${ }^{58}$ et avec tous les autres marginalisés ou exclus qui leur font dénoncer le caractère élitiste de bien des discours, eux-mêmes critiques de la modernité.

Ces divers niveaux de questionnement ouvrent un espace critique qui est celui du lieu des femmes, celui de l'incarnation, du corps, de la sexuation des êtres vivants que nous sommes; mais aussi du lieu des personnes marginalisées ou exclues, que ce soit de par la couleur de peau, leur comportement sexuel, leur santé mentale ou leurs coutumes.

56. Même si d'autres penseurs masculins ont déjà entrepris ce travail, les femmes le font de manière encore plus radicale : $c f$. par exemple G.M. JANTZEN, op. cit.

57. "The power of anger and the work of love", comme dirait Beverly Harrison, dans Union Seminary Quarterly Review 36, 1980-81, p. 49.

58. Je pense aussi aux théologies féministes américaines, la "womanist", la 'mujerista", aussi bien que celles qui viennent d'Asie ou d'Afrique... 
Quand une prise de pouvoir vient de ces lieux, ne provoque-t-elle pas le pouvoir dominant en dévoilant ce qu'il tient pour évident mais qui s'avère une illusion : y aurait-il une vérité s'imposant à tous plutôt qu'une variété de chemins qui constituent un processus vivant? Et ce processus vivant ne serait-il pas habité par un horizon de transcendance auquel nous sommes partie prenante, hommes et femmes? Une tâche importante se dessine pour développer notre pouvoir de création et de transformation, sous le mode de la coopération et de la solidarité, celui du dialogue entre les femmes et les hommes, dans leurs communautés diverses, dialogue qui s'exprimera en une pluralité de « parler-dieu ».

\section{RÉSUMÉ}

En «féminisant " Dieu, la théologie féministe se trouve à confronter le pouvoir masculin et patriarcal comme pouvoir de domination. Dans cet article, nous donnons un aperçu d'études sur le patriarcat, particulièrement dans les religions, afin de cerner l'origine et donc les conditions dans lesquelles le pouvoir patriarcal s'est développé. Puis dans la présentation des discours féministes sur Dieu, dans une perspective critique d'abord, et dans un effort de reconstruction au féminin ensuite, apparaissent des modèles de rapports de pouvoir, tout autant qu'une conception du pouvoir. Un questionnement de fond suit qui engage la poursuite d'une réflexion articulée sur des pratiques de dire Dieue.

\section{ABSTRACT}

When feminist theology "feminizes" "God" it confronts masculine, patriarchal power as the power of domination. This article surveys patriarchy within the context of religion to discover the origin and conditions in which it has developed. It then presents feminist discourses about "God" through a critical perspective ; and finally, in an effort to reconstruct the feminine, it discusses the concept of power and power relationships. Fundamental questions are asked in a reflection about the ways in which the concept of God is expressed. 